\title{
On the feasibility of a channel-dependent scheduling for the SC-FDMA in 3GPP-LTE (mobile environment) based on a prioritized- bifacet Hungarian method
}

\author{
Gerardo Agni Medina-Acosta* and José Antonio Delgado-Penín
}

\begin{abstract}
We propose a methodology based on the prioritization and opportunistic reuse of the optimization algorithm known as Hungarian method for the feasible implementation of a channel-dependent scheduler in the long-term evolution uplink (single carrier frequency division multiple access system). This proposal aims to offer a solution to the third generation system's constraint of allocating only adjacent subcarriers, by providing an optimal resource allotment under a fairness scheme. A multiuser mobile environment following the third generation partnership project TS 45.005v9.3.0/25.943v9.0.0 was also implemented for evaluating the scheduler's performance. From the results, it was possible to examine the channel frequency response for all users (four user equipments) along the whole bandwidth, to visualize the dynamic resource allocation for each of the 10,000 channel realizations considered, to generate the statistical distribution and cumulative distribution functions of the obtained global costs, as well as to evaluate the system's performance once the proposed algorithm was embedded. Comparing and emphasizing the benefits of utilizing the proposed dynamic allotment instead of the classic static-scheduling and other existent methods.
\end{abstract}

Keywords: channel-dependent scheduling, Hungarian method, LTE uplink, multiuser transmission, SC-FDMA; optimal resource allocation, scheduling algorithm

\section{Introduction}

The third Generation Partnership Project (3GPP) has agreed to utilize the single carrier frequency division multiple access (SC-FDMA) as the transmission scheme commissioned to carry out the uplink multiuser access for long-term evolution (LTE). This decision is largely supported because the SC-FDMA preserves most of the main benefits (e.g., multipath mitigation, bandwidth scalability, etc.) given by OFDMA [1,2], while at the same time it adds a key advantage consisting on reducing significantly the variations in the instantaneous power of the transmitted signal [3-7]. This peak-to-average power ratio (PAPR) reduction translates into a direct benefit to the user equipments (UE) mainly in

\footnotetext{
* Correspondence: agni_medina@tsc.upc.edu

Technical University of Catalonia, Barcelona Tech, Signal Theory and Communications Department, Building D4, Campus Nord, Jordi Girona 31,
} Barcelona, 08034, Spain

terms of power consumption. In order to achieve this, the SC-FDMA prepends a discrete Fourier transform (DFT) to the conventional transmission chain of an OFDMA system, which produces that the amplitude on each output subcarrier be a linear combination of all the data symbols that were transmitted in the same time instant, which leads to a virtual or implicit single carrier structure. For this reason, in practice the SC-FDMA system requires that the subcarriers destined to each user be allocated in a contiguous way (i.e., localized mapping), which reduces the flexibility in resource allocation when a dynamic scheduling is considered to be incorporated to the system [8].

When the variety of channel conditions in a multiuser transmission are exploited [9], the dynamic allocation of resources brings significant benefits to the system's performance when each user is allocated to a particular spectrum portion identified as the most the suitable one

reproduction in any medium, provided the original work is properly cited. 
to carry out the communication. In this regard, a methodology for implementing a channel-dependent scheduler based on the prioritization and opportunistic reutilization (maximizing/minimizing) of the algorithm known as Hungarian method is proposed as a feasible solution for the dynamic allocation in LTE uplink. A pre-processing consisting in splitting the bandwidth in a number of segments or resource chunks (RC) equal to the number of users participating in the transmission is required to build the matrix of metrics that is given as input for the proposed algorithm, which aims to provide an optimal resource allocation under a one-by-one or fairness scheme.

This paper is organized as follows. Section 2 presents the mathematical foundations behind the Hungarian method, to later on introducing a step-by-step of the proposed methodology. Section 3 describes the implementation of a multiuser mobile environment according to the normative given by the 3GPP technical specifications. Section 4 shows and discusses in detail the obtained results. Finally, Section 5 summarizes the conclusions and provides an insight about the future work.

\section{Assignment problem methodology}

\subsection{Hungarian method}

The assignment problem described here consists in assigning $n$ tasks to $n$ possible candidates on a one-toone basis in an optimal way. For this purpose, it has to be taken into account that there are exactly $n$ ! ways to assign $n$ tasks to $n$ candidates, and that in order to find the optimal allotment, all $n$ ! combinations would have to be checked until finding the optimal combination providing the minimum global cost (sum of the individual costs).

The Hungarian mathematicians, D. Konig and E. Egervary, proposed an alternative to the computation of all possible combinations (which results computational inefficient as $n$ ! gets larger, e.g., $10 !=3,628,800$ ) through the algorithm known as Hungarian method [10].

The Hungarian method is based on the theorem that is stated below.

Theorem 1: If a constant is added (or subtracted) to every element of any row (or column) of a given $n$-by- $n$ cost matrix in an assignment problem, then the assignment which minimizes the total cost for the new matrix will also minimize the total cost matrix.

In this regard, $C_{i j} \geq 0$ is the cost of assigning the $i$ th candidate to the $j$ th task to build the input cost matrix.

$$
C=\begin{array}{cccc}
C_{1,1} & C_{1,2} & \cdots & C_{1, n} \\
C_{2,1} & C_{2,2} & \cdots & C_{2,1} \\
\vdots & \vdots & & \vdots \\
C_{n, 1} & C_{n, 2} & \cdots & C_{n, n}
\end{array}
$$

Thus, the optimal one-to-one assignment is achieved when the function shown below is minimized.

$$
\text { Optimal_Allotment }=\sum_{i=1}^{n} \sum_{j=1}^{n} c i j A i j \text {. }
$$

where $A_{i j}=1$ if the $i$ th candidate is assigned to the $j$ th task, and $A_{i j}=0$ otherwise.

Once the algorithm's mathematical aspects have been discussed, the procedure outlined by the Hungarian method to find an optimal solution consists of the following steps:

-Step 1: To identify and subtract the minimum number in each row from the entire row.

-Step 2: To identify and subtract the minimum number in each column from the entire column.

-Step 3: Cross all zeros in the matrix with as few lines (horizontal and/or vertical only) as possible.

-Step 4: Test for optimality:

- If the minimum number of covering lines is $n$, an optimal assignment of zeros is possible and we are done.

- If the minimum number of covering lines is less than $\mathrm{n}$, an optimal assignment of zeros is not yet possible. And in this case, is necessary to proceed to Step 5.

-Step 5: To determine the smallest entry not covered by any line. Subtract this entry from each uncovered row, and then add it to each covered column. Return to Step 3.

Thus, the fact of following the previous steps will enable us to solve the assignment problem by obtaining an optimal one-to-one allotment.

\subsection{Strategies for crossing the zeros}

Although the method described before has a welldefined set of steps to follow, what is stipulated in Step 3 turns out to be very open or intuitive. This because even when the minimum number of lines is tried to be utilized, several solutions could take place (there is not a concrete rule to follow for crossing the zeros). Meaning that even when for a particular crossing decision the requirement could be thought as satisfied, an one-toone assignment could not have been found.

On the other hand regarding the test for optimality, the fact that the number of remaining zeros in the resulting matrix be larger than $n$ (as it happens in most of the cases) also leads to an open or intuitive decision about the best zeros selection.

So, largely the inherent intuitive parts of the Hungarian method make it difficult to code. For this reason what is proposed here is to follow two strategies (Row's 
\& Alternating priority strategy) in order to carry out the crossing procedure, highlighting that this proposal is complemented with the methodology described in next section.

\subsubsection{Row's priority strategy}

Under this strategy the priority is given to the horizontal lines over the vertical ones. This means that for the matrix resulting from Step 2 we count the number of zeros per row as well as per column, the values are stored and after a one-by-one comparison (starting with those having the highest population of zeros, or from the first row/column to the last one if they are equal), whenever the number of zeros in a row is found to be greater or equal than the number of zeros in a column, an horizontal line is traced. The row/column traced is crossed or discarded, and the one-by-one comparison is made once again between the remaining values.

An illustrative example detailing the incorporation of this strategy to the Hungarian method is shown in Figure 1.

In the above figure each of the steps dictated by the Hungarian algorithm were exemplified. Highlighting the step 3, because is just there where the row's priority strategy took place. Regarding this step, the numbers placed next to each of the lines indicate the order in which they were traced, while it is mentioned that step 4 is included due that after the last crossing is possible to know if the test for optimality is fulfilled (as happened with the last step 3 or $C$ ) or not. Another point to emphasize in this example has to do with the final stage or the so called zeros selection stage, being the carried out strategy to count the number of zeros per row in order to identify the one having less zeros. This way, the row and column crossing the selected zero are cancelled to later on simply to apply the same logic until finishing. However, for this discrimination process when more than two rows have the same number of zeros, the selection is taken from the first row to the last one.

\subsubsection{Alternating priority strategy}

In this prioritization strategy what is proposed is to alternate the priorities starting with columns. This way at the beginning of the procedure the vertical lines have priority over the horizontal lines until reaching the first test for optimality, and if the process needs to be continued then the priority is switched to the rows. So, this alternation persists until satisfying the criterion enunciated in Step 4 by the Hungarian method. A second illustrative example describing the way this strategy operates is shown in Figure 2.

From the last figure we can observe that, by following a prioritization based on the alternation of columns and rows it is possible to find an optimal solution. Being important to note that in the above example the first and the last step 3 (or in other words A and C) followed a vertical's line prioritization, while the second step 3 (or B) worked under a horizontal priority scheme. Highlighting that when the comparison of zeros resulted to be equal, the crossing strictly follows a prioritization from first to last column/row. However, this hierarchical order also takes into account if there is still a zero to be crossed, this aiming at giving the preference to the next column/row when necessary.

\subsection{Prioritized-bifacet Hungarian method (PBHM)}

As it was mentioned at the beginning of this paper, the dynamic resource allocation plays a crucial role in

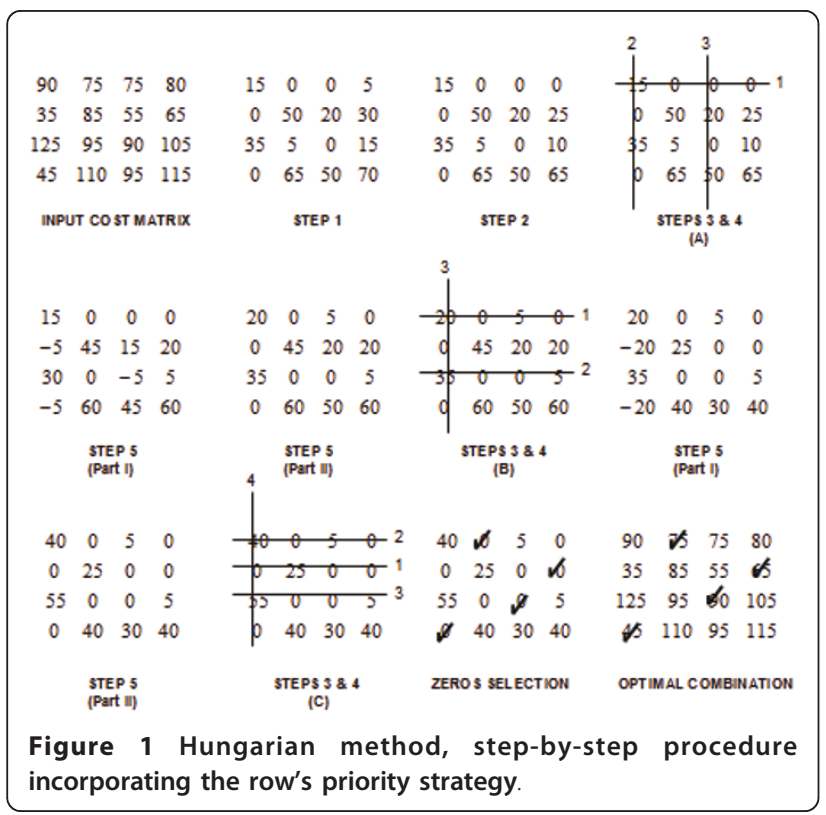

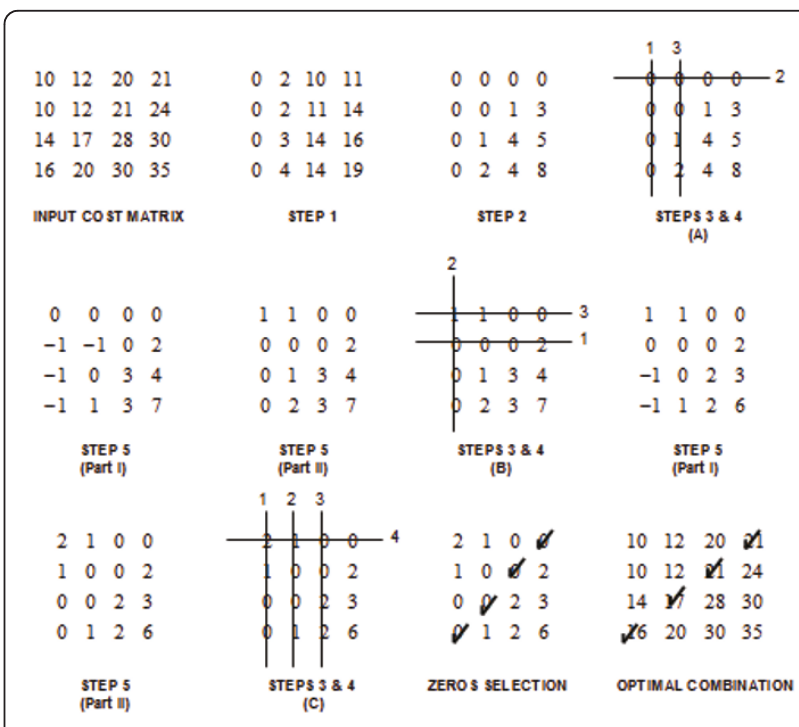

Figure 2 Hungarian method, step-by-step procedure including the alternating priority strategy. 
benefit of a multiuser wireless communications system, where the diversity provided by the channel conditions of each user is utilized as a favorable condition.

For this reason, what is proposed here is a channeldependent scheduler intended to be used in the 3GPPLTE uplink (SC-FDMA system), which is based on the Hungarian algorithm by means of following a methodology aiming at increasing the feasibility of its implementation.

In a broad sense, in order to perform the scheduling the channel state information (CSI) is ideally needed per every transmission time interval (TTI) for all the UEs over the whole bandwidth. Regarding this, although in this paper the CSI is assumed to be perfectly known, it is relevant to mention that 3GPP standard has introduced the transmission of sounding reference signals (SRS) in order to acquire frequency selective information, which provides a realistic alternative to this requirement [11].

In terms of this proposal, the information about the channel conditions provided by all the users is transformed to metrics distributed along the bandwidth, just as happen with other proposals (e.g., search tree-based algorithm). So, these metrics indicate the channel impairments for a number of bandwidth segments (or RC) equal to the number of users to be served, having as an objective to build a metrics matrix (or cost matrix) that can be used as input of the proposed methodology, aiming at find the optimal resource allotment under a fairness principle.

Getting back to the algorithm which constitutes the basis of this proposal, the set of steps given at the beginning of this section are oriented to solve an assignment problem where the minimum (optimal) global cost is found. Nevertheless, in the case of the channel-dependent scheduler the idea is to find the combination of metrics providing the maximum global cost. In this regard, the Hungarian method also offers a solution for this kind of problem by modifying only its first step as:

-Step 1: Subtract the values in each row from the maximum number in the row.

This way is possible to get a variant for the algorithm in order to find the maximum total profit assignment. Thus, in order to make feasible the incorporation of this algorithm as channel scheduler for the uplink into the 3GPP-LTE system, the following methodology is proposed.

-Methodology Step 1: To modify the classic Hungarian method to make it work as an optimal assigner providing the maximal global metric.

-Methodology Step 2: To follow hierarchically the two proposed prioritization strategies (Row's \& Alternating), having finished in this point at best.

-Methodology Step 3: To resort to the classic Hungarian method whenever a solution could not be found, aiming at finding the combination minimizing the global metric.

-Methodology Step 4: To retake the Step 1 giving as input a modified version of the original cost matrix, putting zeros in those metrics which provided the minimum global cost according to Step 3.

-Methodology Step 5: In case that it has not been possible to find an optimal solution, then the Greedy method is utilized to provide a near-optimal solution.

Particularly, the Step 3 in the previously given methodology takes advantage of the existing differences found in the first step of the Hungarian method, which depending on its facet (minimizing/maximizing the global metric) leads to a completely different problem to be solved for the rest of steps that compose the algorithm.

This way, the solution provided by Step 3 (when required), provides an alternative input matrix for Step 1 including more zeros (once the worst values were discarded) contributing hereby to the proper operation of the algorithm in order to find the optimal maximum solution.

\subsection{Algorithms comparison}

Once the methodology corresponding to the proposed channel scheduling was given, a direct comparison with other algorithms working under the same principle of utilizing an input cost matrix is shown in Figure 3.

Although not included in the above figure, the Static scheduling is also part of this family of algorithms. Nevertheless due to its nature, it provides (for most of the cases) the worst performance. In fact for the

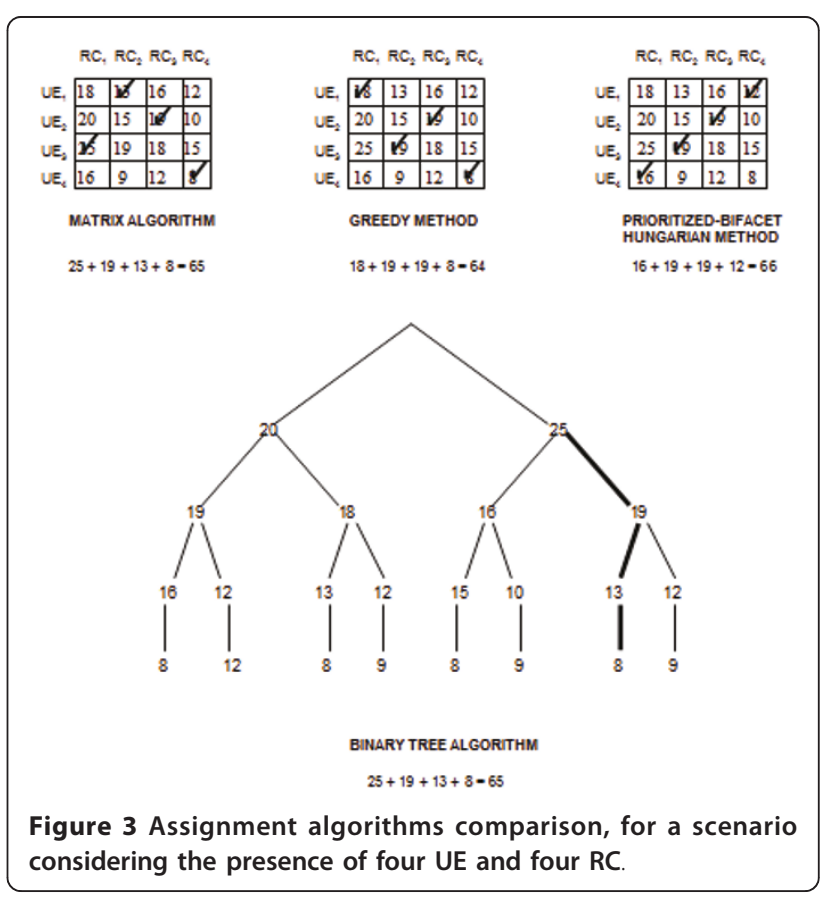


previous example, if a static fashion had been followed by assigning the first $\mathrm{RC}$ to the first $\mathrm{UE}$, the next $\mathrm{RC}$ to the second UE and so on, the global metric would have been equal to 59 .

Regarding the rest of the algorithms [12,13], the socalled Greedy method resulted to be the second worst (global metric $=64$ ) in this example, while the Matrix Algorithm and the Binary Tree Algorithm provided the same solution (or global metric $=65$ ) from a completely different methodology (to keep in mind that for a $4 \times 4$. Cost Matrix there are 1,820 possible combinations). One the other hand, the channel scheduler proposed here (which has been called PBHM because of the methodology it follows) proved to provide an optimal allotment for this problem, maximizing the global metric to a value equal to 66 .

In terms of computational complexity, an asymptotic comparison is shown in Table $1 .^{a}$

The table above describes the complexity order of different algorithms utilized for solving assignment problems, being $n$ equal to the size of the input cost matrix. In this regard, the complexity of the non-optimal Greedy method is reduced per iteration, and its number of operations is estimated as $n / 2(n-1)$ [14]. In the case of the Binary tree, the number of branches is doubled as the matrix size $n$ increases, being the increasing number of nodes equal to $2^{n+1}-1$ [15]. On the other hand and conversely, the complexity of the Hungarian method cannot exactly be determined since it can be classified as a heuristic algorithm (i.e., it involves common sense rules). Nevertheless, James Munkres estimated the maximum number of theoretical operations required as $\left(11 n^{3}+12 n^{2}+31 n\right) / 6$ [16]. So, based on this fact and due that in our proposal the intuitiveness of the original algorithm is overcome by embedding two straightforward strategies and one opportunistic reuse, we can claim that the PBHM has a reduced complexity (however, finding the polynomial is out of the scope of this research work).

\section{Multiuser scenario implementation}

In order to evaluate the performance of the proposed channel-dependent scheduling, four radio channels having characteristics (average delay profiles) according to the dictated by the 3GPP technical specifications 45.005 V9.3.0 (2010-05) were implemented [17]. Concretely, the propagation model which defines the typical case for

Table 1 Asymptotic complexity comparison

\begin{tabular}{ccc}
\hline Algorithm & Complexity & Type \\
\hline Greedy method & $\mathrm{O}\left(n^{2}\right)$ & Quadratic \\
\hline Binary Tree & $\Theta(\log (n))$ & Logarithmic \\
\hline PBHM & $\mathrm{O}\left(\left(11 n^{3}+12 n^{2}+31 n\right) / 6\right)$ & - \\
\hline
\end{tabular}

the urban area was utilized for this purpose. The time delay and the corresponding average powers in the case of a 12 tap configuration are shown in Table 2.

The 3GPP-LTE normative provides for each model (e. g., urban) two alternative equivalent tap settings, in this case the second one is shown in the table above. Regarding this, a discrete channel model was implemented and executed for 10,000 channel realizations, which at the end shown to have a behavior according to the given by the specifications providing the following average powers: $-4.0277,-3.0503,-0.0494,-2.0073,-2.9636$, $-4.9479,-7.0247,-5.0223,-5.9866,-9.0544,-10.9398$, $-9.9556 \mathrm{~dB}$. Set of values that can directly be compared with those given in Table 2 .

So, after it was proved that the random variables ( $\mathrm{r}$. v.'s) involved in the Rayleigh fading process effectively vary according to the specifications, a filtering process which follows the established by the Young model [18] was implemented and incorporated aiming at adding the effects of Doppler shift on the channel. Moreover, given that the 3GPP-LTE system utilizes several bandwidths configurations (e.g., 1.4, 5, or $20 \mathrm{MHz}$ ), an interpolation stage was included in order to get uniformly spaced taps in accordance with the sampling time specified for each configuration [19]. In our case, the frequency domain representation was utilized in order to build the radio channels, being the information about the channel impairments along the whole bandwidth a mandatory requirement to put into operation the scheduler.

Once the foundations regarding the implementation of the wireless channels were given, a set of needed complementary parameters (also extracted from the norm) were chosen in order to simulate and test the proposed channel-dependent scheduler [20-22], which are summarized in Table 3.

The above given parameters constitute the definition of the proposed scenario. The scenario can be described

Table 2 Typical Case for Urban Area: (12 tap setting)

\begin{tabular}{ccc}
\hline Tap number & Relative Time (US) & Average relative power (dB) \\
\hline 1 & 0.0 & -4.0 \\
\hline 2 & 0.2 & -3.0 \\
\hline 3 & 0.4 & 0.0 \\
\hline 4 & 0.6 & -2.0 \\
\hline 5 & 0.8 & -3.0 \\
\hline 6 & 1.2 & -5.0 \\
\hline 7 & 1.4 & -7.0 \\
\hline 8 & 1.8 & -5.0 \\
\hline 9 & 2.4 & -6.0 \\
\hline 10 & 3.0 & -9.0 \\
\hline 11 & 3.2 & -11.0 \\
\hline 12 & 5.0 & -10.0 \\
\hline
\end{tabular}


Table 3 Simulation parameters according to the 3GPP-LTE standard

\begin{tabular}{ccc}
\hline Variable & Value & Description \\
\hline$P$ & 128 & Total number of subcarriers in the system \\
\hline$K$ & 32 & Number of subcarriers per user \\
\hline$Q$ & 4 & Number of simultaneous transmissions (users) without co-channel interference \\
\hline BW & 1.4 & Channel bandwidth $(\mathrm{MHz})$ \\
\hline$T_{\mathrm{s}}$ & 0.52083 & Sampling time $(\mu \mathrm{s})$ \\
\hline UE_speed & $3-120$ & User equipment speed $(\mathrm{km} / \mathrm{h})$ \\
$f_{c}$ & 880 & Carrier frequency $(\mathrm{MHz})$ \\
\hline
\end{tabular}

as a multiple access mobile environment operating at $880 \mathrm{MHz}$ which is characterized by the presence of four users taking place at the same time with speeds between 3 and $120 \mathrm{~km} / \mathrm{h}$, having assigned 32 subcarriers per user after it was assumed that the total of 128 subcarriers (corresponding to a bandwidth equal to $1.4 \mathrm{MHz}$ ) were set available for transmission. It is important to highlight that under normal or real conditions, not all the total number of subcarriers are set available for transmission, because some of them are destined to carry control signals. Nevertheless, in our case no control signals are required because we assume that we perfectly know the channel, being our ultimate goal only to test the channel scheduler suggested.

The generated channels showing the distortions experienced along the whole bandwidth by each of the users, and for the first 20 of 10,000 channel realizations (or transmitted symbols) considered in this analysis, can be observed in Figure 4.

Highlighting that for each of the channels affected by both Rayleigh fading and Doppler shift, the UE speed was considered as a random parameter (in a range from 3 to $120 \mathrm{~km} / \mathrm{h}$ as specified by the 3GPP technical reference 25.943V9.0.0) per user per transmitted symbol, emphasizing that each UE undergoes different channel conditions.

\section{Simulation results}

In the last section, the scenario considered for testing the performance of the proposed scheduler was described. Here, the obtained results are shown and discussed.

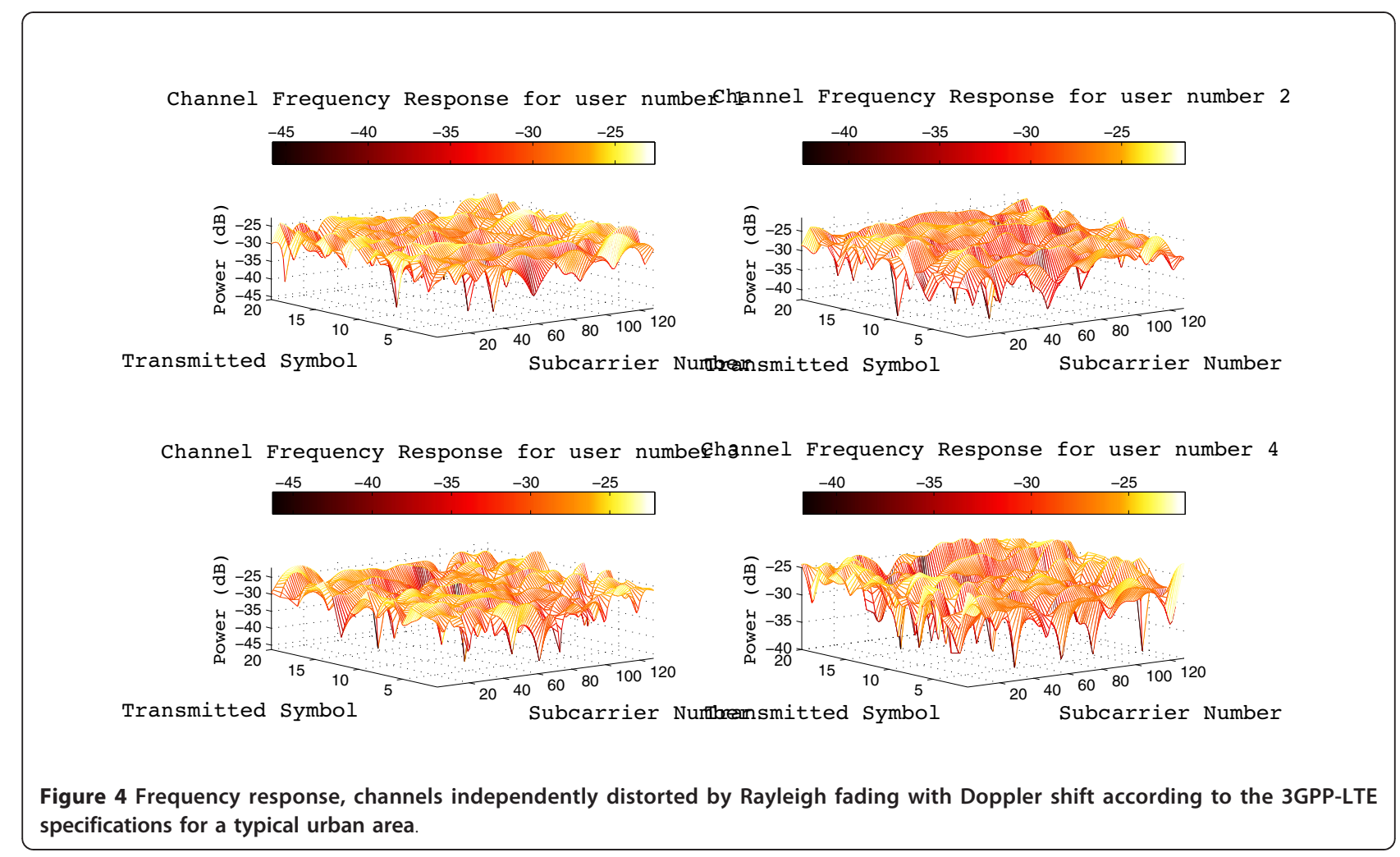


According to the 3GPP-LTE system, the eNode-B is the one that for each TTI (in our case channel realization) acquires the data about the channel conditions for all the users in order to provide this information to the channel scheduler. However, this raw provided information requires a pre-processing stage consisting in segmenting the bandwidth in a number of parts that is equal to the number of UE, to later on computing a metric per segment, aiming at building a matrix of metrics (or Cost Matrix) which is used as input for the channel-dependent scheduler. The dynamic allocation of frequency resources for each of the 10,000 transmitted symbols on the four available RC (bandwidth segments) is shown in Figure 5.

From the above figure, it can be observed the number of times that the algorithm decided (in an optimal way) to allocate each resource chunk to each of the users as function of the impairments found in the channels. In this regard, the identified attenuations per user took values from -22.5934 to $-45.8055 \mathrm{~dB},-21.5074$ to $-42.5226 \mathrm{~dB},-22.1624$ to $-46.2984 \mathrm{~dB},-22.1029$ to $-41.7279 \mathrm{~dB}$. Closely related values, which resulted in a balanced dynamic allocation where all the $\mathrm{RC}$ were utilized to serve the all users, or in other words the whole bandwidth was exploited based on taken the best decisions about the suitability of the wireless channels conditions.

In Figure 6, the global costs (sum of metrics) obtained from each of the allotments made by the proposed algorithm were used to generate a statistical distribution, which was compared with the obtained ones from using other scheduling algorithms.

Roughly speaking, the obtained improvement if a dynamic resource allocation is utilized can be identified by observing a rightward shift of the three last curves with respect to the first one (static), highlighting in this item a compared performance between the search tree algorithm and the proposed method.

Additionally, cumulative distribution functions (CDF) were also generated, which are shown in Figure 7 .

From the figure above, it is possible to have an insight about the probabilities of getting a certain global metric depending on the scheduler in use. For example, in the case of the static scheduling it is possible to observe that there is a probability equal to 0.8 of getting a global cost less or equal to 0.009 , while for the case of the proposed scheduling, the same probability corresponds to a higher global cost which is equal to 0.011. Emphasizing that while the curve corresponding to the matrix algorithm is placed between the upper bound and lower bound given by the static scheduling and PBHM, respectively, the curve belonging to the search tree algorithm can be noted almost superimposed over the lower bound. In this regard, although it may seem that the search tree algorithm and the PBHM could have a similar performance, a very important aspect to consider is that in the case of the first one, as the size of the input matrix increases then the number of branches also increases by two (e.g., $3 \times 3$ matrix $=4$ branches, while a $4 \times 4$

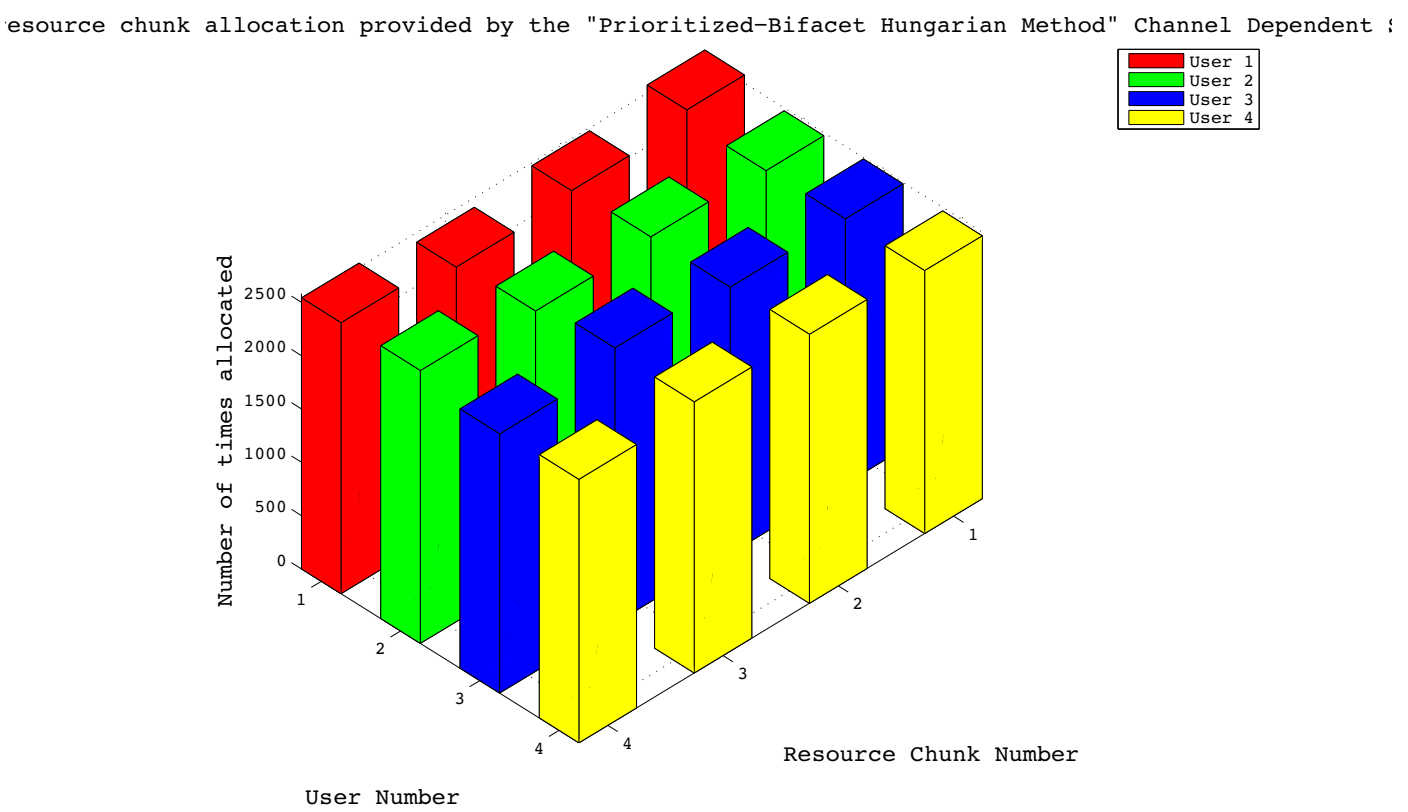

Figure 5 Dynamic allocation per resource chunk after 10,000 transmitted symbols, by considering four simultaneous UEs. 

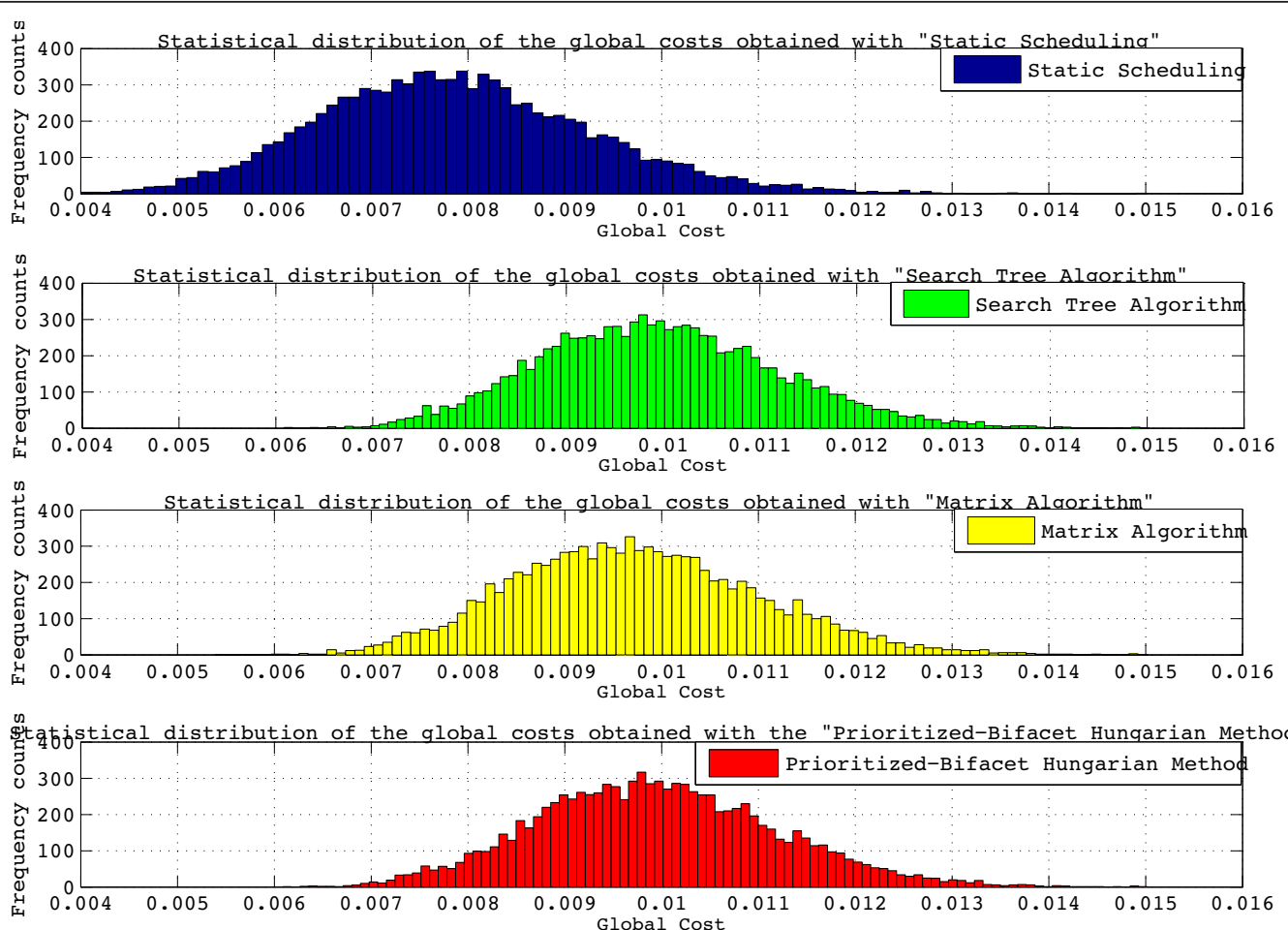

Figure 6 Global metrics, histograms obtained from static and dynamic (several methods) scheduling after 10,000 transmitted symbols.

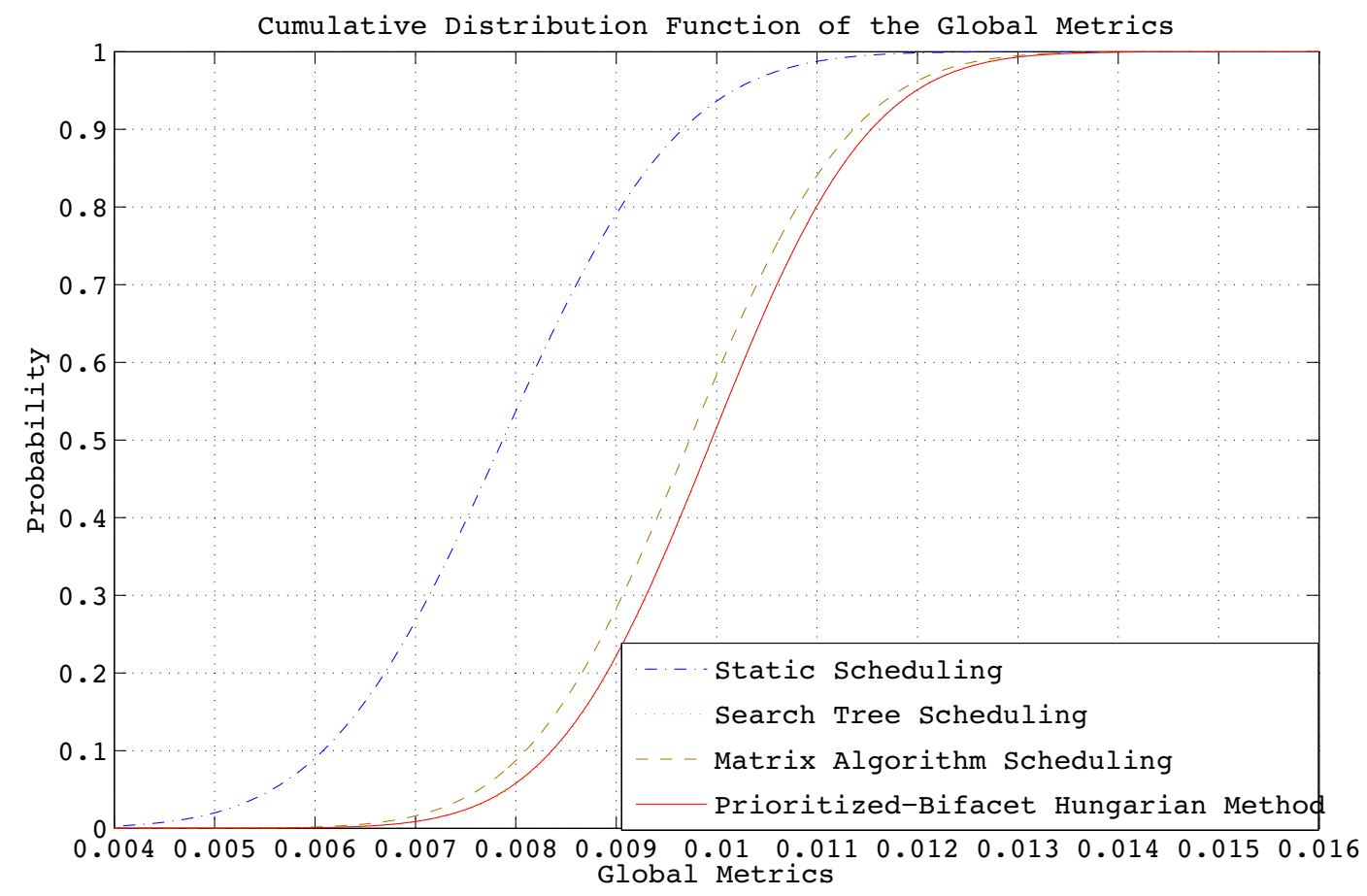

Figure 7 CDFs, static and dynamic (several methods) channel dependent scheduling. 
matrix $=8$ branches), which leads to a problem in terms of implementation feasibility, situation that is tried to be overcome by exploiting the prioritized bi-facet nature of the proposed methodology.

In addition, the specifications shown in Table 3 were utilized for implementing the baseband structure (i.e., Transmitter/Receiver) of a SC-FDMA system aiming at incorporating the proposed channel-dependent scheduler. So, by considering a Q-PSK modulation, a normal cyclic prefix (i.e., 7 SC-FDMA symbols per slot), a set of specific UE speeds $(15,30,60$, and $120 \mathrm{~km} / \mathrm{h}$, respectively, constant), and after the transmission of 10,000 SC-FDMA symbols (i.e., per $E_{b} N_{o}$ ), the system's performance for both the fixed and dynamic allocation of resources is shown in Figure 8.

The bit error rate (BER) curves above allow us to perceive in a clearer manner the benefit of including the proposed algorithm into the system. For example, for an $E_{b} N_{o}$ equal to $12 \mathrm{~dB}$, in the case of the static scheme the system is dealing (in a raw sense) with 1.453 bits having errors per each 10,000 bits received, while when the PBHM is put into operation (i.e., dynamic scheme) the number of errors decreases up to $2.344 / 100,000$. This way, through the system's performance analysis, the strength of the dynamic scheduling over the static one has been emphasized.

Summarizing the proposal, in general it was shown that by following the given methodology it is possible to implement a modified version of the Hungarian method in a feasible way, leading to an alternative to overcome the allocation constraints found in the LTE uplink.

\section{Conclusion}

In this paper, a methodology which applies a double prioritization procedure as well as an opportunistic usage of the two possible facets (maximizer/minimizer) of the optimization algorithm known as Hungarian method was proposed as a feasible solution for the 3GPP-LTE uplink or SC-FDMA system.

The so-called " $P B H M$ " utilizes the knowledge of the variations or distortions undergone by the users that are intended to be served simultaneously per TTI, in order to take a decision about which part of the whole bandwidth is the most suitable (or reliable) to establish a communication by each of the users.

In order to put into operation the proposed algorithm, several mobile radio channels were implemented according to 3GPP-LTE technical specifications for a typical

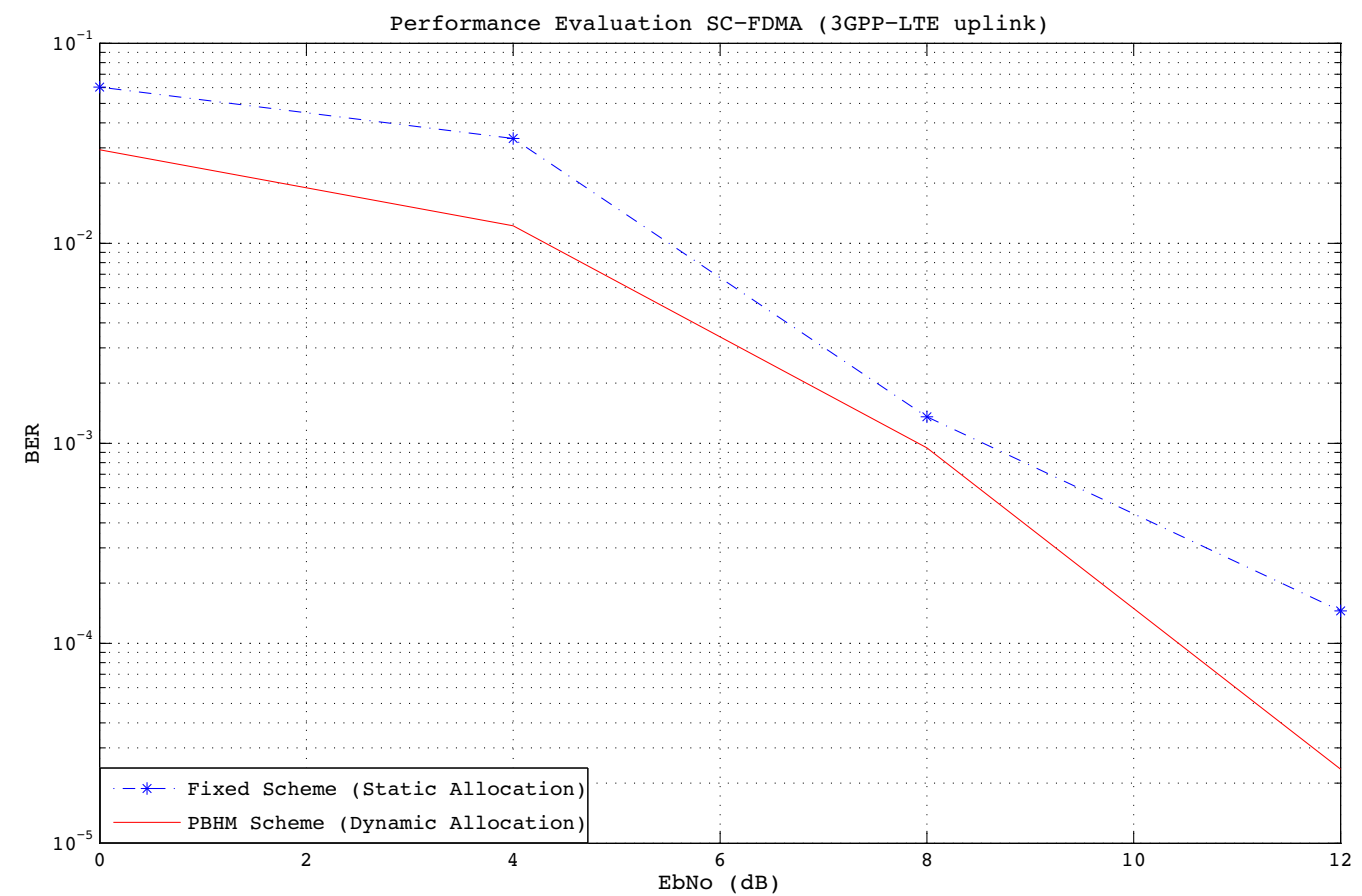

Figure 8 SC-FDMA system, performance evaluation UE 1: static/dynamic allocation of resources. 
urban area. So, once the multiuser environment was created, the channel conditions (frequency domain) for each of the users were extracted by assuming perfect channel knowledge in order to segment the entire bandwidth in a number of $\mathrm{RC}$ matching the number of users, to later on to associate each of them to a specific metric. So, the set of metrics obtained per channel realization were utilized in order to build a square matrix of metrics, which was provided as input of the optimization algorithm. This way, the channel-dependent scheduler aimed to provide the optimal resource allotment by following a fairness fashion, which means that all users were always served under a one-to-one optimal allocation scheme. From the analysis of the obtained results, first it was possible to corroborate that the implemented channels effectively behaved according to the specifications after getting the statistics of the r.v.'s belonging to the Rayleigh fading processes involved. In terms of the proposed channel-dependent scheduler, after 10,000 channel realizations it was possible to count the number of times that each resource chunk was allocated to each user aiming at perceiving the dynamic allocation. Lately, for each of the optimal metrics provided as output of the algorithm, the global metrics were computed with the aim of generating a statistical distribution, which was compared with the obtained ones from other static and dynamic algorithms. Also, empirical CDFs were computed aiming at getting an idea about the probabilities of finding a determined global cost, being the CDF provided by the proposed scheduler the lower bound between the compared algorithms.

Additionally, the baseband structure of the SCFDMA system (Transmiter/Receiver chain) was implemented in order to incorporate the $P B H M$, which allowed us to determine the system's error rate (BER) aiming at perceiving the benefit given by the proposed algorithm.

So, it was proved through mathematical essays as well as by simulating a mobile wireless communication environment that proposed algorithm can be seen as a feasible solution for implementing a channel-dependent scheduling facing the characteristics (contiguous subcarrier allotment) of the 3GPP-LTE uplink system.

\section{Endnote}

${ }^{a}$ In the language of asymptotics (when $n$ is very large) symbols mean. O: grows same rate or slower than, $\Theta$ : same rate, o: grows slower than.

\section{Acknowledgements}

The authors would like to thank the anonymous reviewers for their helpful comments, as well as to the Technical University of Catalonia (UPC), and the
National Council of Science and Technology in Mexico (CONACYT) for the financial support granted.

\section{Competing interests}

The authors declare that they have no competing interests.

Received: 21 September 2010 Accepted: 19 August 2011 Published: 19 August 2011

\section{References}

1. P Man-On, et al. in Multi-Carrier Techniques for Broadband Wireless Communications, vol. 3. (Imperial College Press, London, 2007), p. 17-48

2. M Engels, Wireless OFDM Systems, How to Make them Work? (Kluwer Academic Publishers, 2002), pp. 33-50

3. H Holma, A Toskala, LTE for UMTS OFDMA and SC-FDMA Based Radio Access (Wiley, UK, 2009), pp. 76-80

4. F Khan, LTE for $4 G$ Mobile Broadband, Air Interface Technologies and Performance (Cambridge Press, United Kingdom, 2009), pp. 70-87

5. M Ergen, Mobile Broadband, Including WiMAX and LTE (Springer, New York, 2009), pp. 261-266

6. S Sesia, et al. LTE-The UMTS Long Term Evolution, From Theory to Practice, (Wiley, UK, 2009), pp. 345-357

7. HG Myung, Introduction to single carrier FDMA, $15^{\text {th }}$ European Signal Processing Conference (EUSIPCO) 2007, Poznan, Poland (Sep 2007)

8. Hyung G Myung, Single carrier orthogonal multiple access technique for broadband wireless communications, Ph.D. dissertation, Dept. Elect. \& Comp. USA, Polytechnic Univ., Brooklyn, NY (2007)

9. Andrea Goldsmith, Wireless Communications (Cambridge University Press, 2005), pp. 190-209

10. HW Kuhn, The Hungarian method for the assignment problem. Naval Res. Logistics Quarterly. 2, 83-97 (1955)

11. 3GPP TR 25.814 V7.1.0. Physical Layer Aspects For Evolved UTRA (September 2006)

12. K Keunyoung, et al. Iterative and Greedy Resource Allocation in an uplink OFDMA system, in Proc. 15th IEEE International Symposium on Personal, Indoor and Mobile Radio Communications, (PIMRC, Barcelona, Spain, 2004), pp. 2377-2381

13. FD Calabrese, et al. Search-Tree Based Uplink Channel Aware Packet Scheduling for UTRAN LTE. IEEE Proc. of the 67th Veh. Tech. Conf, 11-14: 1949-1953 (May 2008)

14. Y Kim, J Kim, A 2-D subcarrier allocation scheme for capacity enhancement in a clustered OFDM system. IEICE (Japanese, Institute of Electronics, Information and Communication Engineers) Trans. Commun. E90-B(7), 1880-1883 (1997)

15. TH Cormen, et al. Introduction to Algorithms, 2nd edn. (McGraw-Hill, New York, 2002), pp. 63-66

16. J Munkres, Algorithms for the assignment and transportation problems. Soc. Indus Appl. Math. 5(1), 32-38 (1957)

17. 3GPP TS 45.005 V9.3.0, Radio Access Network: Radio Transmission and Reception (May 2010)

18. DJ Young, NC Beaulieu, The generation of correlated Rayleigh random variates by Inverse Discrete Fourier Transform. IEEE Trans. Commun. 48, 1114-1127 (2000)

19. 3GPP TS 36.101 V9.4.0, User Equipment (UE) radio transmission and reception (June 2010)

20. 3GPP TR 25.814 V7.1.0, Physical Layer aspects for evolved Universal Terrestrial Radio Access (UTRA) (Sept. 2006)

21. 3GPP TS 36.211 V9.1.0, Physical Channels and Modulation (March 2010)

22. 3GPP TR 25.943 V9.0.0, Technical Specification Group Radio Access Network: Deployment aspects (December 2009)

doi:10.1186/1687-1499-2011-71

Cite this article as: Medina-Acosta and Delgado-Penín: On the feasibility of a channel-dependent scheduling for the SC-FDMA in 3GPP-LTE (mobile environment) based on a prioritized-bifacet Hungarian method. EURASIP Journal on Wireless

Communications and Networking 2011 2011:71 